\title{
Subharmonic energy-gap structure and heating effects in superconducting niobium point contacts
}

Flensberg, K.; Hansen, Jørn Bindslev

Published in:

Physical Review B

Link to article, DOI:

10.1103/PhysRevB.40.8693

Publication date:

1989

Document Version

Publisher's PDF, also known as Version of record

Link back to DTU Orbit

Citation (APA):

Flensberg, K., \& Hansen, J. B. (1989). Subharmonic energy-gap structure and heating effects in superconducting niobium point contacts. Physical Review B, 40(13), 8693-8699.

https://doi.org/10.1103/PhysRevB.40.8693

\section{General rights}

Copyright and moral rights for the publications made accessible in the public portal are retained by the authors and/or other copyright owners and it is a condition of accessing publications that users recognise and abide by the legal requirements associated with these rights.

- Users may download and print one copy of any publication from the public portal for the purpose of private study or research.

- You may not further distribute the material or use it for any profit-making activity or commercial gain

- You may freely distribute the URL identifying the publication in the public portal

If you believe that this document breaches copyright please contact us providing details, and we will remove access to the work immediately and investigate your claim. 


\title{
Subharmonic energy-gap structure and heating effects in superconducting niobium point contacts
}

\author{
K. Flensberg and J. Bindslev Hansen \\ Physics Laboratory I, Modellering, Ikke-Lineaer Dynamik og Irreversibel Termodynamik, \\ The Technical University of Denmark, DK-2800 Lyngby, Denmark
}

(Received 26 April 1989)

\begin{abstract}
We present experimental data of the temperature-dependent subharmonic energy-gap structure (SGS) in the current-voltage $(I-V)$ curves of superconducting niobium point contacts. The observed SGS is modified by heating effects. We construct a model of the quasiparticle conductance of metallic superconducting weak links that includes the heating effects self-consistently. Our model is combined with that of Octavio, Blonder, Klapwijk, and Tinkham [Phys. Rev. B 27, 6739 (1983)], which is based on the idea of multiple Andreev scattering in the contact. The shape and the temperature variation of the calculated SGS is found to be in good agreement with the experimental curves for contacts with resistance larger than $5 \Omega$.
\end{abstract}

\section{INTRODUCTION}

Subharmonic energy-gap structure (SGS) is observed in the current-voltage $(I-V)$ characteristics of superconducting metallic weak links (e.g., superconducting point contacts and microbridges) around $V=2 \Delta / n e$, $n=1,2,3, \ldots$ In the same weak links an excess current, $I_{\text {exc }}$, is also seen at high voltage $(V \gg 2 \Delta / e)$. Modeling the superconducting weak link as a superconductor-normal-metal-superconductor (SNS) microconstriction, both of these features of the $I-V$ curve have been successfully explained by Andreev reflections (AR's) at the $N-S$ interfaces. ${ }^{2,3,1}$

The excess current arises from the extra quasiparticle conductance through both $N-S$ interfaces due to AR of the quasiparticles with energy in the superconducting gap region and the accompanying addition of Cooper pairs to the superconductors. Under the assumption that there are no interference effects between the scattering processes at the two interfaces, the total excess current is simply. the sum of the contributions from the two $N-S$ boundaries, but when nonequilibrium effects (which may be induced by AR's) are included, this is no longer correct.,

The SGS is a result of multiple Andreev reflections at the $N-S$ interface. An Andreev reflection is the reflection of an electron as a hole (and the opposite process). A particle gains an energy $2 n e V$ after $n$ Andreev reflections alternating between having electronlike and holelike character and may overcome the energy gap, $2 \Delta$ (for two identical superconductors). This leads to the structures in the $I-V$ curve at $V=2 \Delta / n e$.

For the SNS junctions the experimental verification of this model has only been given on a qualitative level ${ }^{2}$ (in the case of the NS configuration the theory has been justified quantitatively ${ }^{5}$ ). The aim of this work is to make quantitative comparison between theory and experiment for metallic $S-S$ point contacts. In Sec. II we present typical $I-V$ curves and compare them to the results of the Octavio, Blonder, Klapwijk, and Tinkham (OBKT) mod- el. ${ }^{1}$ In Sec. III this model is reviewed, and in order to account for the discrepancies between theory and experiment we incorporate into the model the effect of heating (which is quite large in low- and medium-resistance contacts).

\section{EXPERIMENTAL RESULTS}

The experimental data were obtained by recording $I-V$ and $d V / d I-V$ curves as a function of temperature for a number of point contacts. The study comprised, in all, 65 contacts with normal resistance ranging from 0.1 to 2 $\mathrm{k} \Omega$. The niobium-niobium point contact was chosen for this study due to its three-dimensional geometry and the wide temperature range for niobium accessible in a conventional ${ }^{4} \mathrm{He}$ cryostat. The contact was formed by pressing an electrochemically sharpened wire $(99.5 \% \mathrm{Nb}$ with $l \sim 150 \AA$ ) against a polished flat $(99.99 \% \mathrm{Nb})$. The etching and polishing procedures have been described previously in Refs. 5 and 6 . The $\mathrm{Nb}$ flat was mounted on a temperature-stablized copper block inside a vacuum can immersed in a liquid helium bath at $T=1.3 \mathrm{~K}$. Stainless steel bellows on top of the vacuum can transmitted the mechanical pressure from a differential screw to the contact while only introducing a negligibly small heat leak. This setup is rather unique because it allowed the temperature of the point contact to be varied over the range from 1.3 to $9.2 \mathrm{~K}\left(=T_{c}\right.$ of $\left.\mathrm{Nb}\right)$ without affecting the mechanical setting of the contact.

In Fig. 1 we show experimental data for four representative contacts. Three of the contacts have been compared with theoretical curves calculated from the OKBT model. The elastic scattering $Z$, used in the fit is found from the measured "average" excess current (as will be discussed in Sec. IV, the heating also affects the excess current). The qualitative agreement is reasonable, but in order to understand the quantitative disagreement with regard to the voltage position of the SGS, we have constructed a heating model. 


\section{SGS MODEL INCLUDING HEATING}

In order to see how heating can be incorporated into the SGS model, we first review the basic ideas for the superconductor-isolator-normal-metal-isolator-superconductor (SINIS) configuration as developed by Blonder, Klapwijk, and Tinkham (BKT) (Ref. 3) and Octavio and BKT (OBKT) (Ref. 1) and recently reconsidered by Flensberg, Hansen, and Octavio. ${ }^{4}$

\section{A. The OBKT model}

The theory is restricted to the quasiparticle current, and for this purpose the Bogliubov-deGennes equations were used to derive expressions for the transmission and reflection coefficients for an incoming electron at an NIS interface. Here $I$ is a layer which causes elastic scatter-

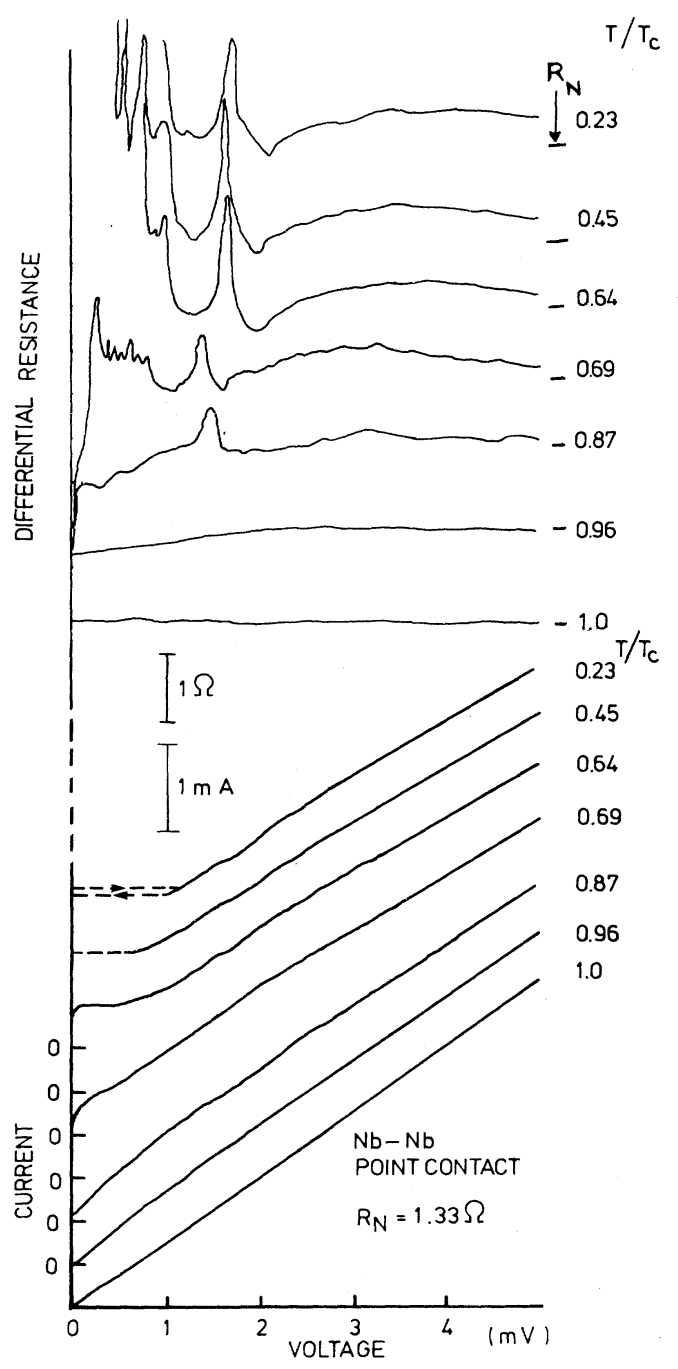

ing. In the BKT model it was modeled by a delta function centered at the NS boundary. The Bogoliubov-de Gennes equations were solved in this geometry, with the pair potential modeled by a step function: zero in the normal metal and constant in superconductor. This gives the transmission coefficient $C(E)$, the normal reflection coefficient $B(E)$, and the Andreev reflection coefficient $A(E)$, where $E$ is the energy of the incoming electron. These probability amplitudes can now be used to derive equations for the nonequilibrium electron distribution function in the normal layer of the SINIS sandwich. Following OBKT, we have at the left interface

$$
\begin{aligned}
f_{\rightarrow}(E)= & C(E) f_{0}(E)+A(E)\left[1-f_{\leftarrow}(-E)\right] \\
& +B(E) f_{\leftarrow}(E) .
\end{aligned}
$$

The arrows correspond to left- and right-going parti-

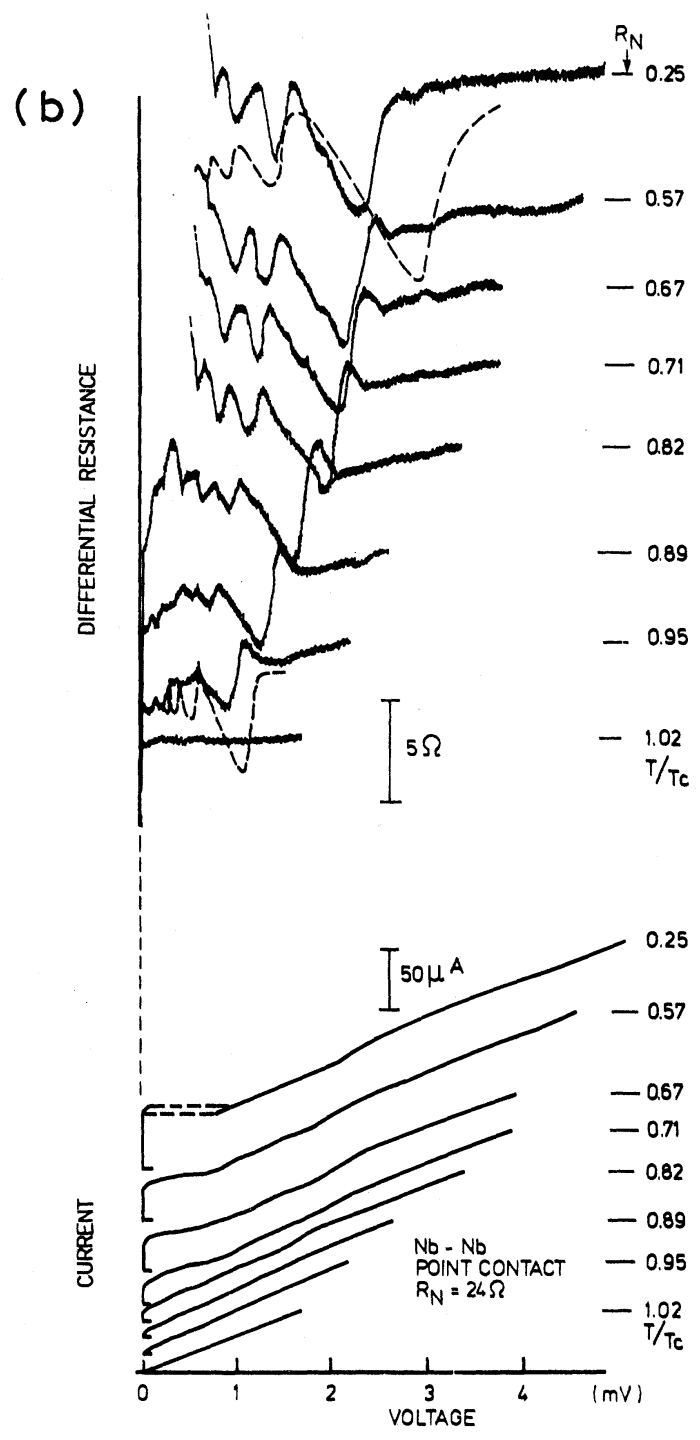

FIG. 1. Experimental data for four different contacts, (a), (b), (c), and (d). For three of the contacts the curves have been compared with theoretical curves based on the OBKT model without heating. The $Z$ value has been determined from the measured excess current in the contacts. 
cles. $f_{0}(E)$ is the Fermi function, $f_{0}(E) C(E)$ is the distribution of the electrons transmitted from the left superconductor to the normal region, and $A(E)\left[1-f_{\leftarrow}(-E)\right]$ is the distribution of left-going holes being Andreev reflected and converted into electrons. The last term in (1) is the distribution of the normal reflected electrons. A similar formula gives the rightgoing distribution at the right end of the normal electrode, and these two are combined through the requirement of particle conservation. In this (one-dimensional) model the potential drops from zero to $-e V$ in the normal region so

$$
f_{\rightleftarrows}(E, 0)=f_{\rightleftarrows}(E+e V, L) .
$$

Equation (1) and the similar equation for $f_{\leftarrow}(E, L)$ together with Eq. (2) may be combined ${ }^{4}$ to give the following equation for $f_{\rightarrow}(E, 0)$ :

$$
\begin{aligned}
f_{\rightarrow}(E)= & A(E) f_{\rightarrow}(E-e V) \\
& +B(E)\left[1-f_{\rightarrow}(-E-e V)\right]+C(E) f_{0}(E),
\end{aligned}
$$

(c)

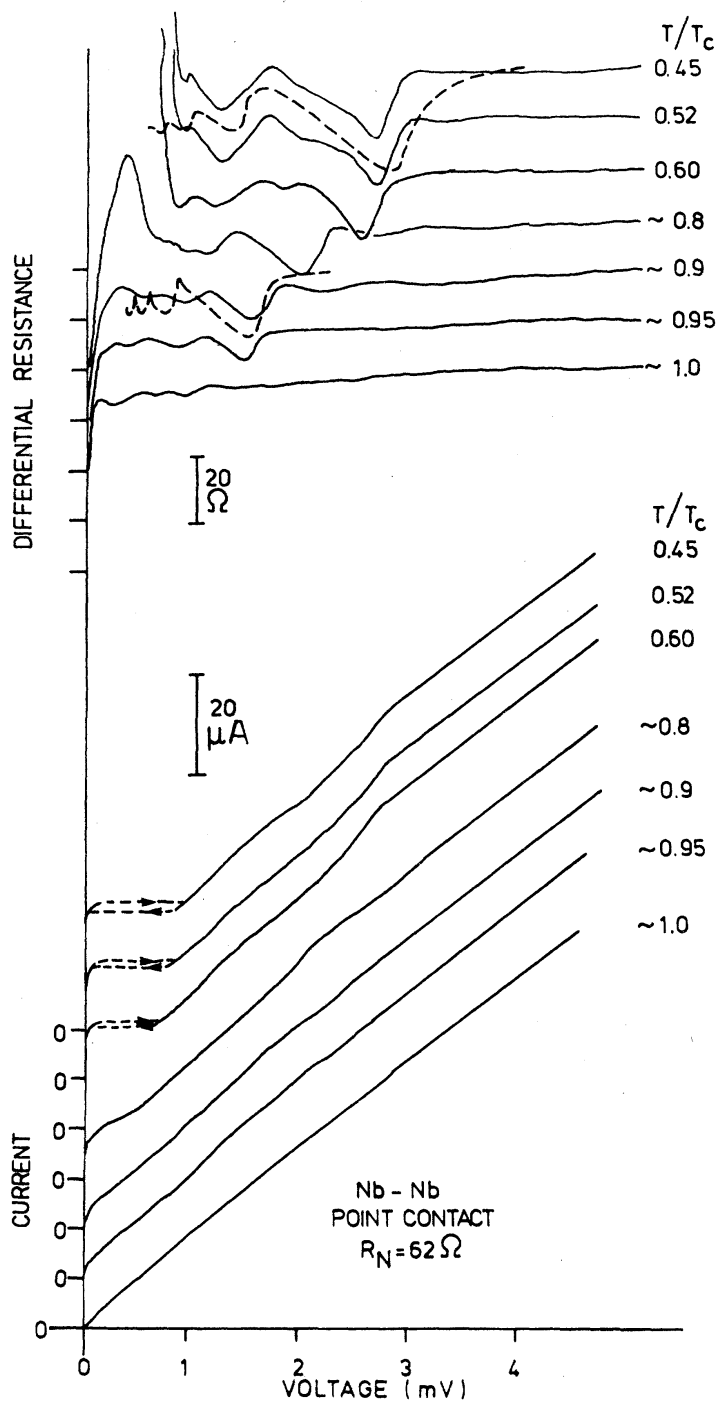

where $f_{\rightarrow}$ has to be computed numerically in a selfconsistent way.

Knowing $f_{\rightarrow}(E, 0), f_{\leftarrow}(E, L)$ is obtained by the relation $^{4}$

$$
f_{\leftarrow}(E)=1-f_{\rightarrow}(-E-e V) .
$$

The net quasiparticle current is found by integrating the difference between the distribution functions for rightand left-going particles:

$$
I_{q}=\frac{1}{e R_{N}} \int_{-\infty}^{\infty} d E\left[f_{\rightarrow}(E)-f_{\leftarrow}(E)\right] .
$$

It is easy to show that

$$
R_{N}=\left(1+2 Z^{2}\right) /\left[2 \mathcal{A} v_{F} e^{2} N(0)\right] ;
$$

$\mathcal{A}$ is the contact area, $v_{F}$ is the Fermi velocity, $N(0)$ the density of states at the Fermi level, and $Z$ is a dimensionless scattering parameter $Z=h_{0} / \hbar v_{F}$ proportional to the strength $h_{0}$, of the $\delta$-function barrier. With the distribution functions in hand, the current is calculated by nu-

(d)

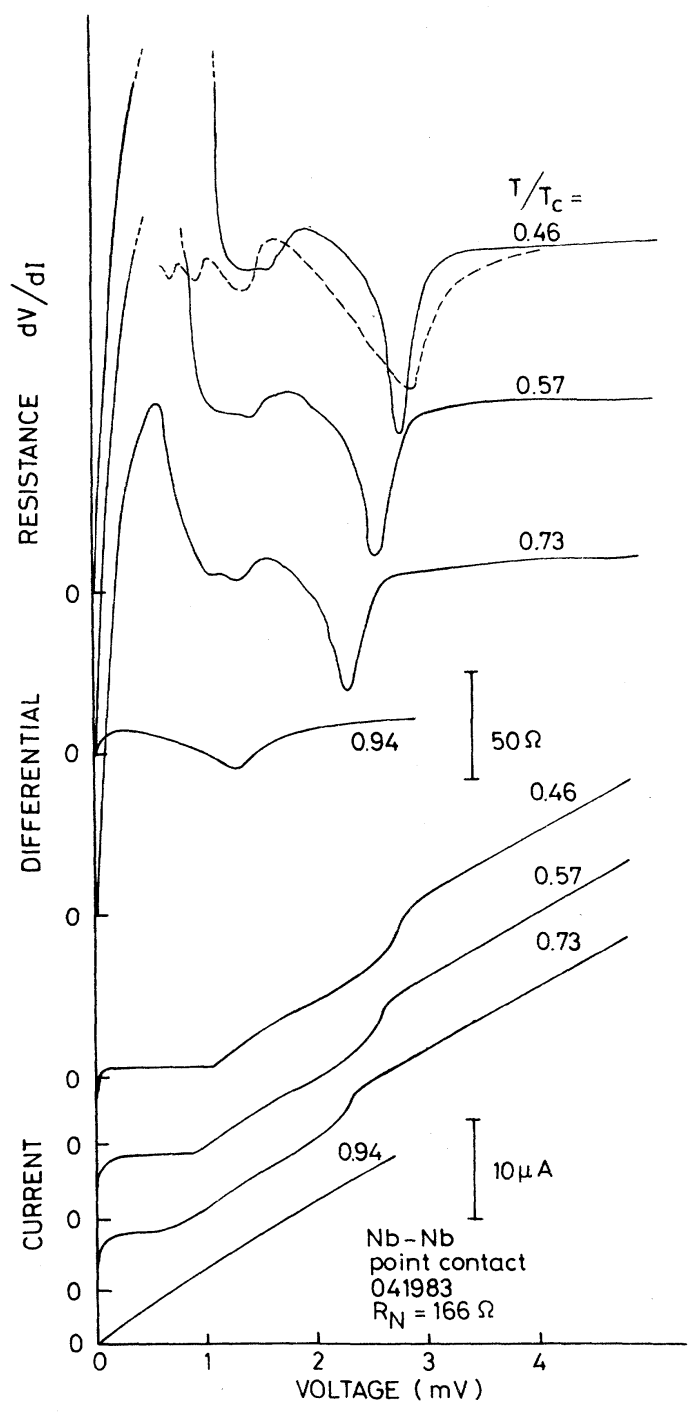

FIG. 1. (Continued). 
merical integration of these [Eq. (5)], and also the derivative $d V / d I_{q}$ can be determined.

\section{B. Heating model}

The effect of heating in the contact is to change the scattering amplitudes, $A, B$, and $C$, through the temperature-induced suppression of the gap parameter. To find this gap suppression we first calculate the temperature profile in the contact area. We model the contact as a three-dimensional cone. Tinkham et al. ${ }^{7}$ have studied the heating effects in a hyperbolic neck and explicitly included the boundary scattering. Without any detailed knowledge of the actual point geometry, we have adopted some of their results since we are only interested in the effects of the heating at some distance from the neck, where the details should be of no importance.

If size effects are taken into account, the effective conductivity is position dependent:

$$
\rho(r)=\rho_{0}(1+l / r),
$$

where $l$ is the electronic mean free path, $r$ is the distance from the center of the neck, and $\rho_{0}$ is the bulk conductivity. The power dissipated in the volume $a<r^{\prime}<r$ is

$$
P(r)=\Omega \int_{a}^{r} d r^{\prime} r^{\prime 2} \rho\left(r^{\prime}\right) i\left(r^{\prime}\right)^{2},
$$

where $a$ is taken to be the radius of the neck. The temperature gradient may then be found from

$$
\frac{d T}{d r}=-\frac{P(r)}{\Omega \kappa(T) r^{2}},
$$

where $\Omega$ is the solid angle as seen from the contact vertex and $\kappa$ is the thermal conductivity.

To calculate $P(r)$ we assume that the current density is given by

$$
i(r)=I_{\text {tot }} / \Omega r^{2}
$$

which implies that no quasiparticle current is converted to supercurrent over the length scale of interest. This assumption is valid for a three-dimensional contact since the charge-imbalance relaxation length $\lambda_{Q^{*}}$ (Ref. 8) is much larger than $l$, and hence the solution to the threedimensional diffusion problem, $\exp \left(-r / \lambda_{Q^{*}}\right) / \Omega r$, is dominated by the $1 / r$ dependence. The cooling of the contact is, however, strongly dependent on the thermal conductivity of the superconductor.

When an electron is Andreev reflected, a pair is injected into the condensate. In more detail, this process is an injection of a pair of quasiparticles decaying over a length $\xi(T)$. For $E<\Delta$ the solution to the Bogoliubov-de Gennes equations has an imaginary part

$$
\operatorname{Im}(k)=h v_{F} / \Delta(T),
$$

and hence the temperature dependence of the reflection and transmission coefficients $A(E), B(E)$, and $C(E)$ via $\Delta(T)$ is determined by $T=T(\xi)$. Octavio et al. ${ }^{9}$ showed that the suppression of the subharmonic energy gap structure scales like $P / \xi(T)$, which is consistent with these arguments.

With the use of Eq. (9), integration of Eqs. (7) and (8) gives an equation for $T(r)$. Tinkham et al. ${ }^{7}$ used the normal-state conductance as given by the WiedemannFranz law

$$
\kappa^{N}=\frac{\pi^{2}}{3}\left(k_{B} / e\right)^{2} T / \rho .
$$

The $\kappa \sim T$ dependence is a good approximation near the critical temperature where the quasiparticle population is large $[f(E) \sim f(\varepsilon)]$, but at low temperatures the conductance is poor since only the excitations contribute to $\kappa_{\text {electron}}$. Since we are interested in the detailed positions of the SGS peaks, we need to use a better value for $\kappa$. We will use the relaxation time approximation of the Bolzmann equation to evaluate the thermal conductivity. The energy current is given by (neglecting thermoelectric effects)

$$
\begin{aligned}
j_{E} & =N(0) \int_{-\infty}^{\infty} d \varepsilon f(E) v(E) E \\
& =-\kappa^{S} \nabla T
\end{aligned}
$$

with the usual notation

$$
E=\left(\varepsilon^{2}+\Delta^{2}\right)^{1 / 2} \text {. }
$$

We get for $\kappa^{S}$

$$
\begin{aligned}
\kappa^{S}= & \sigma^{N} \frac{2}{e^{2} T} \int_{\Delta}^{\infty} d E\left[-\frac{\partial f_{0}(E)}{\partial E}\right] E^{2} N_{S}^{-1}(E) \\
= & \kappa^{N} \frac{3}{2 \pi^{2}}\left[\frac{\Delta}{k_{B} T}\right]^{3} \\
& \times \int_{1}^{\infty} d \xi \xi\left(\xi^{2}-1\right)^{1 / 2} \operatorname{sech}^{2}\left(\xi \Delta / 2 k_{B} T\right),
\end{aligned}
$$

where $\sigma^{N}$ is defined as $\tau_{\mathrm{el}} v_{F}^{2} N(0) e^{2}$. This result for $\kappa^{S}$ is in fact identical to the expression derived by Kadanoff and Martin ${ }^{10}$ based on a full Green's function calculation. We see that at very low temperatures $\kappa^{S}$ decreases exponentially. When $\Delta=0$, Eq. (12) reduces to Eq. (10). In Fig. 2 the heat conductance, as given by Eq. (12), is plot-

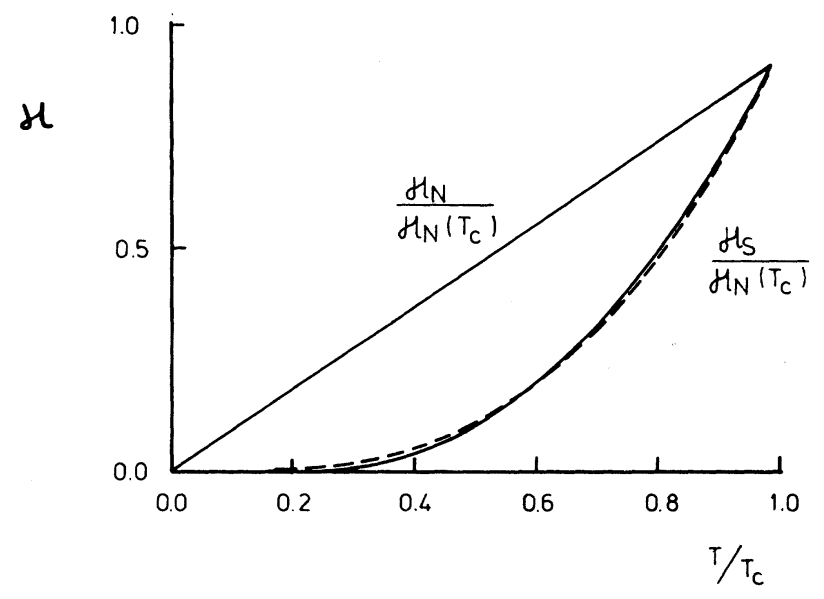

FIG. 2. Electronic thermal conductivity in the superconducting and normal phases (normalized to the conductivity at $T_{c}$ ) vs $T$. The dashed curve is the approximation $\kappa^{S} \sim\left(T / T_{c}\right)^{3}$ used in our model. 
ted versus the reduced temperature $T / T_{c}$. We used the following approximation, also shown in the figure,

$$
\kappa^{S}(T)=\kappa^{N}\left(T_{c}\right)\left(T / T_{c}\right)^{3},
$$

to integrate Eq. (8).

We assume that the contact is in the Sharvin limit, i.e., $l>a$, which is consistent with the fact that many medium- to low-resistance point contacts exhibit excess current. The magnitude of the excess current corresponds to low values of $Z$, indicating a relatively clean metallic contact. For a low ohmic contact (but still satisfying $R_{N}>\rho l / 4 a^{2}$, the Sharvin resistance limit for $Z=0$ ), a low value of $Z$ is an indication that the contact is in the $l>a$ limit.

Integration of Eq. (8) with the use of Eqs. (6), (7), and (9) yields (for $r \gg a$ )

$$
T(r)=T_{0}\left(1+\frac{6 e^{2} T_{c}^{2} R_{N} I_{\text {tot }}^{2} \rho_{0}}{\pi^{2} k_{B}^{2} \Omega r T_{0}^{4}}\right]^{1 / 4},
$$

where we have used

$$
R_{N} \sim 2 \rho_{0}(1+l / 2 a) / \Omega a .
$$

Using

$$
\xi=\xi_{0} / \sqrt{1-T / T_{c}}
$$

and $I_{\text {tot }} R_{N}=V$, Eq. (14) becomes

$T(\xi)=T_{0}\left[1+\alpha\left(R_{N}\right)\left[\frac{T_{c}}{T_{0}}\right]^{4}\left[\frac{e V}{\Delta_{0}}\right]^{2} \sqrt{1-T(\xi) / T_{c}}\right]^{1 / 4}$,

where

$$
\alpha\left(R_{N}\right)=\left(\frac{\Delta_{0}}{k_{B} T}\right)^{2} \frac{6 \rho_{0}}{\pi^{2} \xi_{0} \Omega R_{N}} .
$$

We have solved Eq. (15) numerically under the condition that $e V=2 \Delta(T)$. This gives the deviation from the equilibrium gap, $\Delta_{\mathrm{BCS}}(T)$, due to the effect of heating, at the $e V=2 \Delta(T)$ structure in the $I-V$ curve. This is illustrated in Fig. 3.

The heating parameter $\alpha$ is given by the material parameters [Eq. (16)]. Inserting $\Omega=2 \pi$ and typical values for dirty $\mathrm{Nb}$,

$$
2 \Delta_{0} / k_{B} T_{c}=3.8
$$

and

$$
\rho_{0}=7 \times 10^{-8}
$$

in $\Omega \mathrm{m}$, measured, and using the reduced coherence length as given by

$$
1 / \xi_{0}=1 / \xi_{0, \text { clean }}+1 / l
$$

with $l=15 \mathrm{~nm}$ from the Chambers's relation

$$
\rho l=1 \times 10^{-15} \Omega \mathrm{m}^{2},
$$

we get

$$
\xi_{0}=11 \mathrm{~nm},
$$

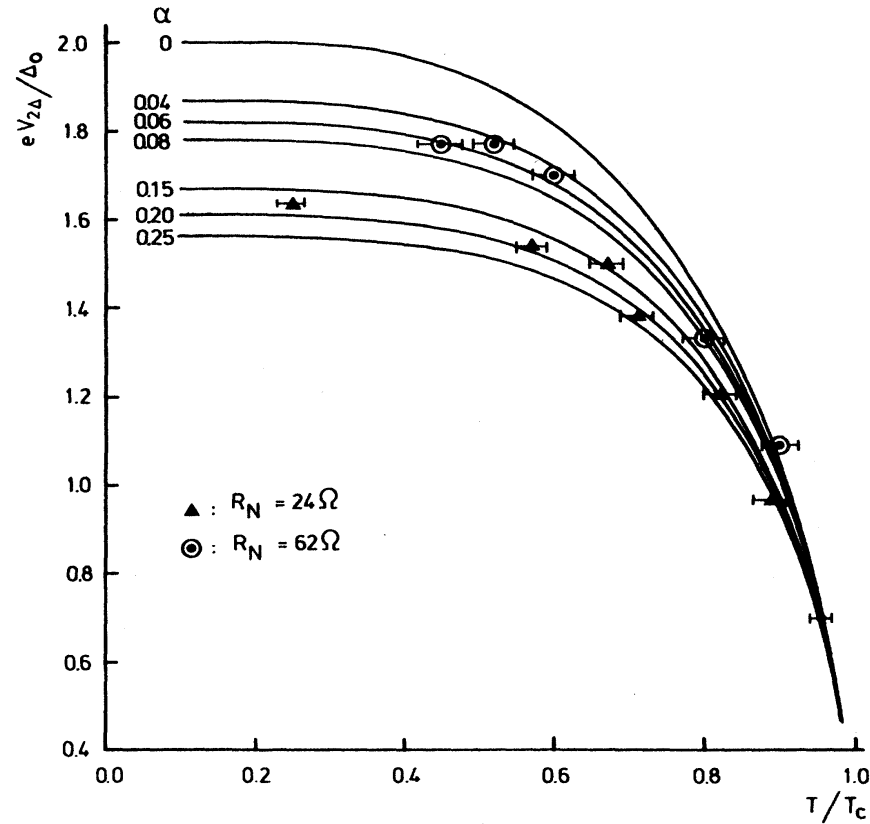

FIG. 3. The temperature dependence of the $2 \Delta$ structure for different values of the heating parameter $\alpha$. Experimental data are plotted in order to determine $\alpha$ for the two contacts shown later in Fig. 5. We take $\alpha=0.20$ for the $R_{N}=24 \Omega$ contact and $\alpha=0.06$ for $R_{N}=62 \Omega$.

and $\alpha$ is then

$$
\alpha\left(R_{N}\right)=2.24 \Omega / R_{N} .
$$

For two of the contacts in Fig. 1 this estimate yields $\alpha=0.09$ (for the $R_{N}=24 \Omega$ contact) and $\alpha=0.04$ (for $R_{N}=62 \Omega$ ). However, in the following we have chosen to determine $\alpha$ (and $Z$ ) by a more realistic fitting procedure.

First an $\alpha$ value is obtained from an $e V(2 \Delta)$ versus $T$ curve (Fig. 3). The order of magnitude for the best fit for $\alpha$ is in agreement with Eq. (20), although this result is not expected to be quantitatively correct. Second, with fixed $\alpha, Z$ is obtained from the measured excess current. In the 1D model all elastic scattering is assumed to take place at the interface. The model is here applied to a $3 \mathrm{D}$ contact with elastic volume scattering. We therefore regard $Z$ as a purely phenomological parameter. Figure 4 shows $I_{\text {exc }}$ versus temperature for different values of $Z$, and this gives for the $R_{N}=24 \Omega$ contact $Z=0.65$, and $Z=0.60$ for $R_{N}=24 \Omega$. In this way the two free parameters $\alpha$ and $Z$ are determined from the experimental data, i.e., from the normal-state resistance $R_{N}$, the excess current, and the gap suppression. The quantitative comparison with theory for the two contacts is shown in Figs. $5(a)$ and $5(b)$. The theoretical data in these figures are calculated on the basis of the OBKT theory combined with the gap suppression found from $\Delta_{B C S}(T)$ with the temperature given by Eq. (15).

There is an overall good agreement in the magnitude of the gap structures as seen in the $d V / d I$ versus $I$ curve and also in their voltage position. The shape of the struc- 


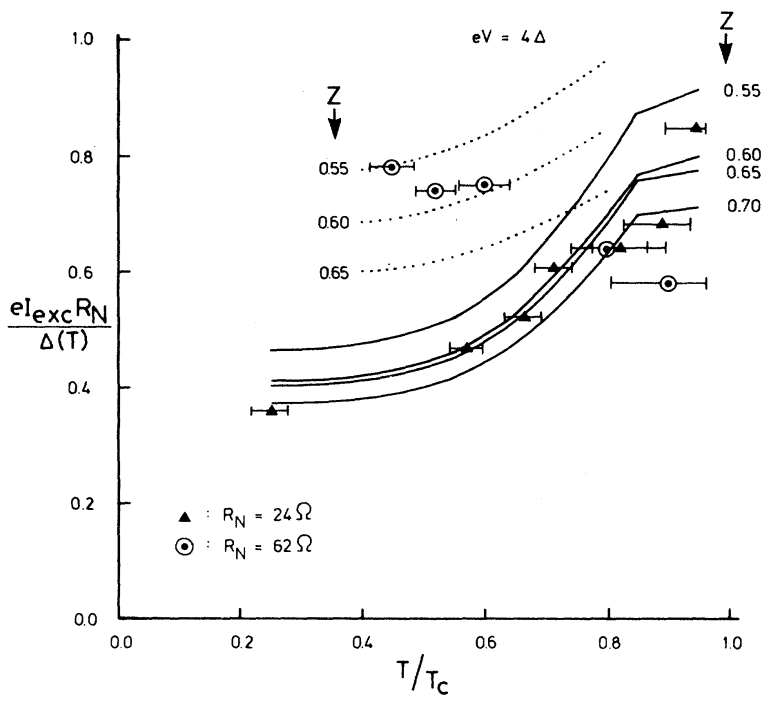

FIG. 4. Normalized excess current vs temperature for $Z$ in the region $0.55<Z<0.70$, and for two different values of the heating parameter, $\alpha=0.06$ (dashed lines) and $\alpha=0.20$ (solid lines). These curves are used in the fitting procedure. Inclusion of the heating effect in the theory makes the excess current temperature dependent.

tures is well reproduced by the theoretical curves. However, the subharmonic structures are dominated by the background "lift" of the differential resistance due to the Josephson effect. In the resistively shunted junction model (with zero capacitance) the dynamical resistance is given by

$$
d V / d I=R_{N}\left[1+\left(I_{C} R_{N} / V\right)^{2}\right]^{1 / 2},
$$

and this is roughly in agreement with the experimental $d V / d I$ versus $V$ curves in Fig. 5 for $V>\hbar / e \tau_{\text {inel }} \sim 200$ $\mu \mathrm{V}$. To deal with the Josephson effect the following differential equation should be solved:

$$
I_{C} \sin \phi+I_{q} \frac{\hbar}{2 e} \frac{d \phi}{d t}=I
$$

where $I_{q}$ is only known numerically. Since $I_{q}$ differs only a little from $I_{q}=\left\langle R_{q}\right\rangle V$, we have tried to use the above expression multiplied by the calculated $d V / d I_{q}$ :

$$
d V / d I=\left(d V / d I_{q}\right)\left[1+\left(I_{C}\left\langle R_{q}\right\rangle / V\right)^{2}\right]^{1 / 2}
$$

and found improved agreement with the experimental data at low voltages (using the measured critical current).

We have not succeeded in making a fit to the point contact in Fig. 1(a) which has a low resistance $\left(R_{N}=1.3\right.$ $\Omega$ ). This contact is representative of the low-resistance contact data. The contacts with $R_{N}$ larger than $5 \Omega$, however, typically exhibited characteristics like the ones shown for the two contacts in Figs. 5(a) and 5(b).

The model presented here seems adequate to account for the heating effect on the SGS at low and medium voltages. However, with increasing voltage the temperature in the neck rises, and when $T_{c}$ is reached the normal region expands. Consequently, the region where the gap is (a)

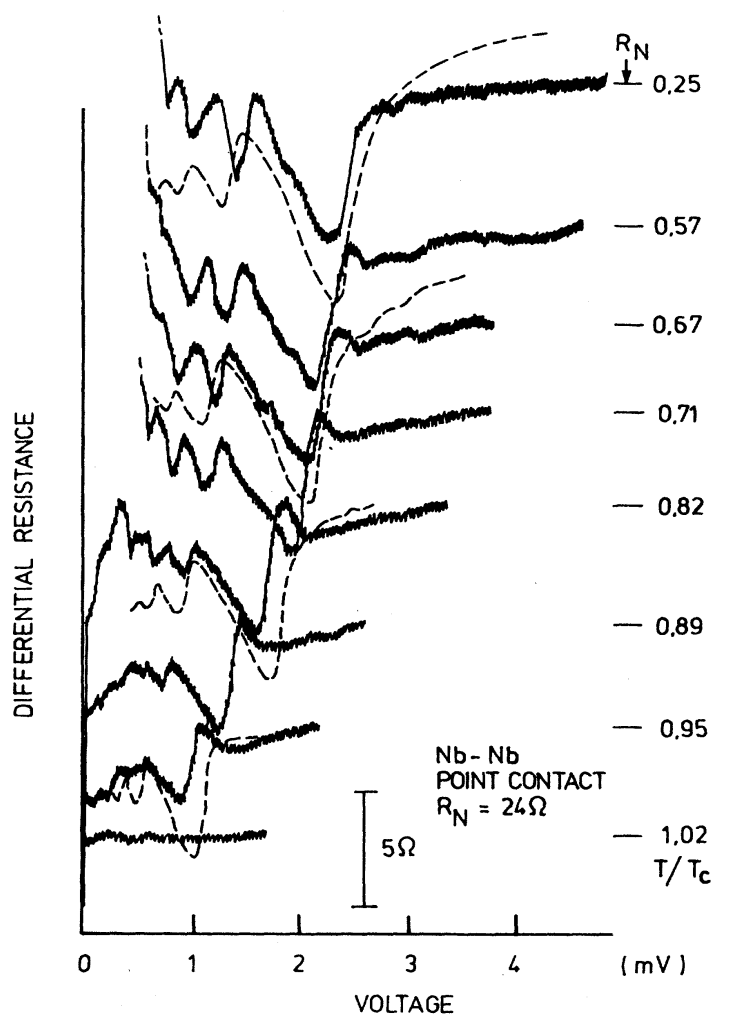

(b)

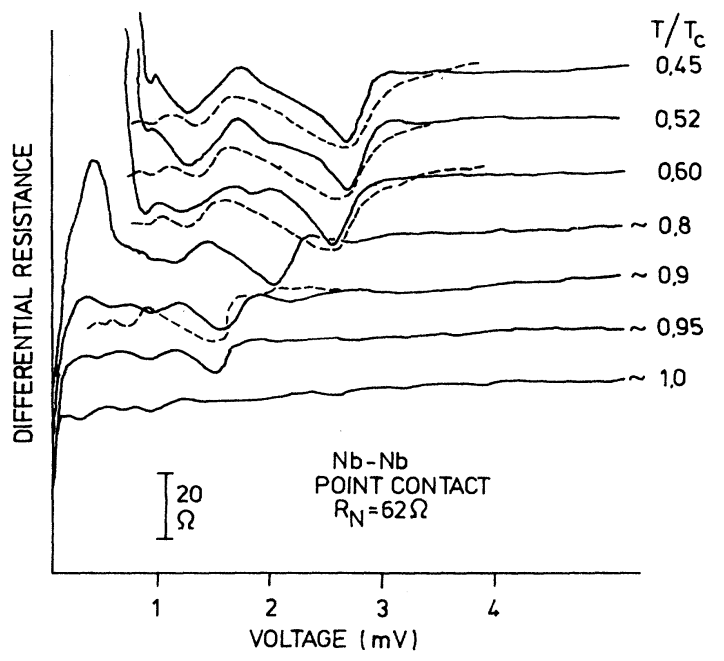

FIG. 5. Experimental data for two of the point contacts in Fig. 1. Theoretical curves (dashed) based on the OBKT theory with the heating model presented here are shown (at the selected temperatures). 
probed by the incident quasiparticles moves out into the electrodes. This leads to a better cooling of that region. When a balance between the heat production and this effect of self-heating is reached, the excess current will be constant. The experimental $I-V$ curves indicate that the heating effects saturate for voltages above the $2 \Delta / e$ structure. The expansion of the normal region could be included in the model without introducing any new parameters. Instead of $\xi(T)$ in Eq. (15) we would have a new effective distance, $\xi_{\text {eff }}$, over which the gap is probed. Assuming that $a<\xi$, we have

$$
\xi_{\text {eff }}(T)=\xi(T)+r_{\text {int }}(T)
$$

where $r_{\text {int }}(T)$ is the distance from the center of the contact to the interface. Using Eq. (14), this distance is given implicitly by $T\left(r_{\text {int }}\right)=T_{c}$. Since we are here mainly interested in the details of the SGS we have not carried this idea through explicitly.

\section{CONCLUSION}

We have compared typical experimental $d I / d V-V$ curves of niobium point contacts (at various tempera- tures) and the theory of SGS developed by OBKT. The theoretical $d V / d I$ versus $V$ curves have qualitative features like the experimental data for contacts with $R_{N}$ larger than $5 \Omega$ presented in this paper and also experimental data for micro-constrictions reported in the literature. ${ }^{11,12}$ We have included the effect of heating in the theoretical model in the case of a point contact. With this improvement, which takes into account the fact that the electronic heat conductance decreases very rapidly for temperatures below $T_{c}$, we are able to explain in a simple way the suppression of the energy gap for all temperatures. There is, however, room for further improvement of the model: The Josephson effect and the voltage-dependent expansion of the normal region could be included.

\section{ACKNOWLEDGMENTS}

We thank M. Octavio, T. Klapwijk, and M. Tinkham for fruitful discussions. One of us (J.B.H.) would like to thank the Harvard Superconductivity Group for help and hospitality during the course of the experimental work reported here.
${ }^{1}$ M. Octavio, G. E. Blonder, M. Tinkham, and T. M. Klapwijk, Phys. Rev. B 27, 6739 (1983).

${ }^{2}$ T. M. Klapwijk, G. E. Blonder, and M. Tinkham, Physica 109-110 B\&C, 1657 (1982).

${ }^{3}$ G. E. Blonder, M. Tinkham, and T. M. Klapwijk, Phys. Rev. B 25, 4515 (1982).

${ }^{4}$ K. Flensberg, J. Bindslev Hansen, and M. Octavio, Phys. Rev. B 38, 8707 (1988).

${ }^{5}$ G. E. Blonder and M. Tinkham, Phys. Rev. B 27, 112 (1983).

${ }^{6} \mathrm{~J}$. Bindslev Hansen, M. Tinkham, and M. Octavio, in Proceedings of the 17th International Low Temperature Conference, edited by Eckern et al. (Elsevier, Amsterdam, 1984), p. 435.
${ }^{7}$ M. Tinkham, M. Octavio, and W. J. Skocpol, J. Appl. Phys. 48, 1311 (1977).

${ }^{8}$ See, e.g., Nonequilibrium Superconductivity, Phonons and $\mathrm{Ka}$ pitza Boundaries, edited by K. E. Gray (Plenum, New York, 1981).

${ }^{9}$ M. Octavio, W. J. Skocpol, and M. Tinkham, IEEE Trans. Magn. MAG-13, 739 (1977).

${ }^{10}$ L. P. Kadanoff and P. C. Martin, Phys. Rev. 124, 670 (1961).

${ }^{11}$ Yu. Ya. Divin and F. Ya. Nad, J. Phys. C 6, 599 (1978).

${ }^{12}$ P. E. Gregers-Hansen, E. Hendriks, and M. T. Levinsen, Phys. Rev. Lett 31, 524 (1973). 